\title{
Evolución de la Unidad Económica Agrícola en la zona núcleo agrícola de la Argentina en el quinquenio 2015 - 2020
}

DOI: $10.46932 / \mathrm{sfjdv2n1-060}$

Received in: November 1st, 2020

Accepted in: December 30th, 2020

\author{
Mg. Ing. Agr. Porstmann Juan Carlos \\ Facultad de Ciencias Agrarias. Universidad Nacional de Rosario. \\ Campo Experimental Villarino CC No 14. (S2125ZAA) Zavalla - Santa Fe ARGENTINA. \\ E-mail: jcporstmann@yahoo.com.ar \\ Mg. CPN e Ing. Agr. Mancini Carina \\ Facultad de Ciencias Agrarias. Universidad Nacional de Rosario \\ Campo Experimental Villarino CC No 14. (S2125ZAA) Zavalla - Santa Fe ARGENTINA. \\ E-mail: cmancini@fcecon.unr.edu.ar \\ Ing. Agr. Rivera Rúa Victorina \\ Facultad de Ciencias Agrarias. Universidad Nacional de Rosario \\ Campo Experimental Villarino CC No 14. (S2125ZAA) Zavalla - Santa Fe ARGENTINA. \\ E-mail: victorinarr@yahoo.com.ar \\ Ing. Agr. Ercoli Rubén \\ Facultad de Ciencias Agrarias. Universidad Nacional de Rosario \\ Campo Experimental Villarino CC No 14. (S2125ZAA) Zavalla - Santa Fe ARGENTINA. \\ E-mail: raercoli1@yahoo.com.ar \\ Mg. Ing. Agr. Vigna Cecilia \\ Facultad de Ciencias Agrarias. Universidad Nacional de Rosario \\ Campo Experimental Villarino CC No 14. (S2125ZAA) Zavalla - Santa Fe ARGENTINA. \\ E-mail: cvigna@unr.edu.ar \\ Ing. Agr. Wagner Agustín \\ Facultad de Ciencias Agrarias. Universidad Nacional de Rosario \\ Campo Experimental Villarino CC No 14. (S2125ZAA) Zavalla - Santa Fe ARGENTINA. \\ E-mail: aguswagner008@gmail.com
}

\section{RESUMEN}

El concepto de Unidad Económica Agropecuaria (U.E.A.) se refiere a la superficie trabajada que permite vivir y progresar económicamente a una familia tipo. A lo largo de los años, y analizando los censos agropecuarios de las últimas décadas, se observa una tendencia, en todo nuestro país, a la concentración de la superficie en un número cada vez menor de empresas agropecuarias. Este fenómeno puede ser explicado a través de la determinación de la U.E.A. El objetivo del trabajo es calcular la U.E.A. de las explotaciones agrícolas de los departamentos del sur de la Provincia de Santa Fe de la zona núcleo y analizar las causas de sus variaciones para la serie 2015/2020. Se emplea el Método de Urbano Fernández que es el utilizado a nivel oficial en la región. Los resultados obtenidos muestran que la superficie promedio de la U.E.A. para el quinquenio es $\mathbf{3 5 8 , 3} \mathbf{h a}^{\mathbf{1}}$, significativamente superior a la superficie modal del área en estudio (sur de Santa $\mathrm{Fe}$ ) que fue estimada en $\mathbf{1 3 5 , 4}$ ha.

Palabras claves: unidad económica agrícola; agricultura pampeana; resultados económicos; escala de producción.

\footnotetext{
${ }^{1}$ ha significa hectáreas. Su equivalente en el Sistema Internacional de Unidades (SI) es $10.000 \mathrm{~m}^{2}$
} 


\section{INTRODUCCIÓN}

En la zona núcleo agrícola de la Región Pampeana Argentina, durante las últimas dos décadas se consolidó un proceso de expansión de la frontera agrícola, impulsado principalmente por el cultivo de soja. Esto transformó la región en un área mayoritariamente agrícola, con un reducido número de actividades que se caracterizan por la presencia predominante de cultivos extensivos tales como maíz, trigo, soja de primera siembra y de segunda siembra (Porstmann y López 2001).

Este proceso de agriculturización impulsado por el precio de los granos y el incremento de productividad agrícola, indujo a una alta competencia por el uso de la tierra entre las actividades agrícolas y las ganaderas, provocando el desplazamiento de la ganadería a tierras de menor capacidad productiva para la agricultura (Porstmann et al., 2013).

En forma simultánea, estas transformaciones de la agricultura pampeana, se han caracterizado por la presencia de ciclos económicos de expansión y contracción, a partir de diversos factores como el precio de los granos, de la tierra, el avance tecnológico y su impacto en la productividad y los costos; los derechos de exportación, el tipo de cambio, la presión fiscal y los ciclos de inflación de precios entre otros. La incidencia negativa impacta en el resultado económico de las empresas, de tal forma que es necesaria una mayor superficie trabajada o escala de producción para cubrir los costos y generar crecimiento.

El concepto de Unidad Económica Agrícola (U.E.A.) está definido en la Legislación Agraria Argentina en el art. 21 de la Ley 14.392 y se refiere a "todo predio que por su superficie, calidad de tierra, ubicación, mejoras y demás condiciones de explotación, racionalmente trabajado por una familia agraria que aporte la mayor parte del trabajo necesario, permita subvenir a sus necesidades y a una evolución favorable de la empresa en el mediano plazo.

El antecedente de la definición de U.E.A. es la ley de colonización 12.636 del año 1940 cuya finalidad era poblar el interior del país, aumentando el número de agricultores propietarios, dividiendo los campos en unidades económicas familiares (García Galilea 2016).

Actualmente, según Bono (2013) el cálculo de la U.E.A. ha tomado protagonismo como herramienta para el Ordenamiento Territorial Rural para garantizar un desarrollo sustentable de la producción agrícola, frente al proceso de subdivisión y comercialización de la tierra.

La U.E.A. es un indicador de tamaño o superficie mínima, medido en hectáreas, que contribuye a caracterizar los sistemas predominantes de una región y brinda información que permite la comparación con otras regiones, otros sistemas productivos y otros niveles de productividad. Así mismo, si se analizan valores históricos de U.E.A., determinados anualmente en una serie de tiempo, puede observarse la evolución del sector agrario en su ambiente, en un contexto atravesado por variables físico-biológicas, tecnológicas, climáticas, socio-culturales, económicas y de mercado entre otras. En un contexto desfavorable se espera que la superficie de U.E.A. sea mayor en comparación con aquellos escenarios favorables. 
La superficie modal de las explotaciones agropecuarias (EAPs) de la región en estudio se incrementó en los últimos 30 años pasando de 125,0 ha a 135,4 ha según datos del último Censo Agropecuario Nacional (CNA2008).

En un estudio previo, Porstmann y López (2001) establecen la importancia del factor precio de los granos sobre el tamaño de la U.E.A., determinando una superficie promedio de U.E.A. de 232,0 ha para la serie histórica 1992-2001 en el sur de la provincia de Santa Fe.

Esta investigación parte de la hipótesis de que este proceso de transformación de la estructura agraria responde en parte a estos factores económicos que en su condición negativa conducen a la necesidad de trabajar una mayor superficie anual, verificándose una tendencia creciente de la U.E.A. en la región.

En este sentido, la rentabilidad de la agricultura y su relación con la escala mínima de producción, ha sido durante las últimas décadas, una preocupación constante que ha dado lugar a fuertes reclamos sectoriales. Al mismo tiempo ha sido tema de estudio y debate en el diseño de políticas agrarias. En virtud de ello este trabajo pretende aportar una visión sobre el grado de evolución económica de la agricultura pampeana a través de la determinación de la Unidad Económica Agrícola, brindando información sobre los factores que mayor incidencia han tenido sobre el desempeño económico de las empresas representativas de la región pampeana núcleo.

El objetivo de este trabajo es calcular la U.E.A. de las explotaciones agrícolas de los departamentos del sur de la Provincia de Santa Fe de la zona núcleo y analizar las causas más relevantes de sus variaciones para la serie 2015/2020. Se presentan, en este trabajo, las UEAs de las campañas 2015/2016, 2016/2017, 2017/2018, 2018/2019 у 2019/2020.

\section{METODOLOGÍA}

El cálculo de la superficie de la U.E.A. debe reflejar las condiciones predominantes de la zona, tales como el uso del suelo, su productividad, la tecnología empleada, la distancia a los mercados, etc. para llegar a la determinación de una unidad de explotación que sea representativa del área en estudio.

Para realizar el análisis económico de la explotación se debe fijar primeramente el tamaño más frecuente de superficie (modal), a lo que se le incorporará la dotación y composición del capital promedio de la zona y se aplicará la tecnología predominante del área y los rendimientos promedios.

El Método de Urbano Fernández es el utilizado a nivel oficial. Es fundamentalmente gráfico, pero puede resolverse analíticamente. Se basa en la estimación previa de la superficie modal, a partir de la cual se determinan los ingresos y los costos de la misma. Tomando esta superficie se estructura una explotación hipotética y razonada. La superficie de la Unidad Económica surge de la intersección de la recta de ingresos y la parábola de costos.

Para el cálculo de costo de producción, como señala Zuliani Susana (2017), se suma la renta fundiaria 
de la explotación, que considera el $4 \%$ del valor de la tierra, el interés del capital del 5\% del valor de las mejoras, el 6\% del capital fijo, un $8 \%$ del capital circulante, amortizaciones de las mejoras y del capital de explotación fijo vivo e inanimado, gastos variables y fijos, incluyendo la remuneración del trabajo del productor.

También tiene en cuenta un Fondo de Previsión y reserva, que depende del riesgo del tipo de explotación, variando entre 10 y $30 \%$ sobre la totalidad de los puntos anteriores.

Serán: $10 \%$ en explotación de cría

$12-15 \%$ en agricultura

$18-20 \%$ en tambo

20-25\% en explotaciones intensivas

$30 \%$ en vivero

En síntesis, los Costos Totales están determinados por la suma de:

a. Gastos Variables: labores de implantación y protección, insumos, cosecha, comercialización y seguro de granizo.

b. Gastos Fijos: conservación de mejoras, comunicaciones, movilidad, honorarios, impuestos fijos, tasas, seguridad social y gastos generales.

c. Amortizaciones del capital mejoras y maquinarias.

d. Trabajo del Productor.

e. Costos de Oportunidad del capital total

f. Fondo de Previsión y Reserva para empresas agrícolas del $12 \%$

Con respecto a los Ingresos Totales, para cada uno de los cultivos sembrados en la campaña se determina la superficie, el rendimiento y el precio de mercado en el mes de cosecha (neto de derechos de exportación o "retenciones").

Los costos de producción y los ingresos varían en función de la superficie. Los costos están representados geométricamente por una parábola y los ingresos por una recta de pendiente positiva.

Analíticamente se tiene:

Parabola de costos: $y^{2}=2 p * x$

Siendo $2 \mathrm{p}=$ constante $\mathrm{y} x=$ superficie

Recta de ingresos: $z=b * x$

Siendo $b=$ Ingreso Bruto por unidad de superficie y $\mathrm{x}$ la superficie

Siguiendo el desarrollo de González y Pagliettini (1993), se igualan costos e ingresos y luego se 
despeja superficie, siendo:

$$
\mathrm{UEA}=\left({\frac{\text { costo }}{\text { ingreso }^{2}}}^{2}\right) * \text { superficie modal }
$$

La superficie modal se estimó con los datos sobre número de EAPs por rango de superficie en propiedad del Censo Nacional Agropecuario 2008, correspondientes a los siete departamentos de la provincia de Santa Fe incluidos en esta región (departamentos Belgrano, Caseros, Constitución, General López, Iriondo, Rosario y San Lorenzo).

Tabla No 1:Determinación de la superficie modal. Sur de Santa Fe.

Escala de Extensión (ha)

\begin{tabular}{lrrrrr}
\hline Rango ha & $\mathbf{2 5 , 1}$ a 50 & $\mathbf{5 0 , 1}$ a 100 & $\mathbf{1 0 0 , 1}$ a 200 & $\mathbf{2 0 0 , 1 ~ a ~ 5 0 0}$ & Total \\
\hline $\mathrm{N}^{\mathbf{o}}$ EAPs & $1.203,0$ & $2.572,0$ & $2.109,0$ & $1.419,0$ & $7.303,0$ \\
\hline Ha por estratato & $45.346,5$ & $190.206,0$ & $302.837,2$ & $450.449,3$ & $988.839,0$ \\
\hline & & & \multicolumn{2}{c}{ Supreficie Modal en ha: } & $\mathbf{1 3 5 , 4 0}$ \\
\cline { 5 - 6 }
\end{tabular}

\section{RESULTADOS}

En primer lugar se presenta el uso del suelo, representado por los cultivos o actividades más frecuentes de la campaña. Su participación relativa en el área de estudio y el rendimiento de cada actividad se determina a partir de la información elaborada por el Ministerio de Agroindustria (2020). Las actividades Trigo y Soja de 2da. conocidas como “doble cultivo" se realizan sobre la misma superficie en la campaña.

Como se puede observar la participación de los cultivos es bastante estable en el período considerado, siendo la Soja de 1ra la de mayor incidencia.

Tabla $N^{\circ} 1$ Superficie modal, uso del suelo e Ingreso Bruto anual por campaña

\begin{tabular}{|c|c|c|c|c|c|c|c|c|c|}
\hline \multicolumn{5}{|c|}{ CAMPAÑA 2015/2016 } & \multicolumn{5}{|c|}{ CAMPAÑA 2016/2017 } \\
\hline \multicolumn{2}{|c|}{ Superficie modal en ha: } & \multicolumn{2}{|l|}{135,40} & \multirow{2}{*}{$\begin{array}{l}\text { Ingreso Bruto } \\
\text { Total USD } \\
\end{array}$} & \multicolumn{2}{|c|}{ Superficie modal en ha: } & \multicolumn{2}{|l|}{135,40} & \multirow{2}{*}{$\begin{array}{l}\text { Ingreso Bruto } \\
\text { Total USD } \\
\end{array}$} \\
\hline Actividades & Parcipación & Hectáreas & q/ha & & Actividades & Parcipación & Hectáreas & $\mathrm{q} / \mathrm{ha}$ & \\
\hline Soja de 1ra & $75,5 \%$ & 102,16 & 31,98 & $96.067,6$ & Soja de $1 \mathrm{ra}$ & $59,2 \%$ & 80,14 & 38,57 & $73.568,9$ \\
\hline Maíz & $13,0 \%$ & 17,61 & 87,00 & $29.935,7$ & Maíz & $19,7 \%$ & 26,62 & 87,29 & $35.438,6$ \\
\hline Trigo & $11,5 \%$ & 15,63 & 36,43 & $7.537,8$ & Trigo & $21,2 \%$ & 28,64 & 40,29 & $16.892,7$ \\
\hline \multirow[t]{2}{*}{ Soja de 2da } & $11,5 \%$ & 15,63 & 24,83 & $11.410,5$ & Soja de 2da & $21,2 \%$ & 28,64 & 29,95 & $20.416,5$ \\
\hline & $111,5 \%$ & 151,0 & & $144.951,6$ & & $121,2 \%$ & 164,0 & & $146.316,8$ \\
\hline
\end{tabular}




\begin{tabular}{|c|c|c|c|c|}
\hline \multicolumn{5}{|c|}{ CAMPAÑA 2017/2018 } \\
\hline \multicolumn{2}{|c|}{ Superficie modal en ha: } & \multicolumn{2}{|l|}{135,40} & Ingreso Bruto \\
\hline Actividades & Parcipación & Hectáreas & $\mathrm{q} / \mathrm{ha}$ & Total USD \\
\hline Soja de $1 \mathrm{ra}$ & $61,2 \%$ & 82,87 & 27,38 & $63.318,4$ \\
\hline Maíz & $21,1 \%$ & 28,62 & 78,00 & $41.851,6$ \\
\hline Trigo & $17,7 \%$ & 23,91 & 42,01 & $16.064,4$ \\
\hline \multirow[t]{2}{*}{ Soja de 2da } & $17,7 \%$ & 23,91 & 21,26 & $14.185,5$ \\
\hline & $117,7 \%$ & 159,3 & & $135.419,8$ \\
\hline
\end{tabular}

\begin{tabular}{cccrc}
\hline \multicolumn{5}{c}{ CAMPAÑA 2018/2019 } \\
\hline Superficie modal en ha: & $\mathbf{1 3 5 , 4 0}$ & & Ingreso Bruto \\
\hline Actividades & Parcipación & Hectáreas & q/ha & Total USD \\
\hline Soja de 1ra & $58,7 \%$ & 79,47 & 41,39 & $72.361,4$ \\
\hline Maíz & $17,6 \%$ & 23,79 & 102,50 & $34.133,5$ \\
\hline Trigo & $23,7 \%$ & 32,14 & 29,86 & $19.098,5$ \\
\hline Soja de 2da & $23,7 \%$ & 32,14 & 32,14 & $22.725,0$ \\
\hline \multicolumn{7}{c}{$123,7 \%$} & 167,5 & & $\mathbf{1 4 8 . 3 1 8 , 4}$ \\
\hline
\end{tabular}

\begin{tabular}{|c|c|c|c|c|}
\hline \multicolumn{5}{|c|}{ CAMPAÑA 2019/2020 } \\
\hline \multicolumn{2}{|c|}{ Superficie modal en ha: } & \multicolumn{2}{|l|}{135,40} & Ingreso Bruto \\
\hline Actividades & Parcipación & Hectáreas & $\mathrm{q} / \mathrm{ha}$ & Total USD \\
\hline Soja de 1ra & $50,8 \%$ & 68,84 & 36,84 & $56.291,4$ \\
\hline Maíz & $21,3 \%$ & 28,87 & 107,00 & $37.074,5$ \\
\hline Trigo & $27,8 \%$ & 37,69 & 39,35 & $29.511,5$ \\
\hline \multirow[t]{2}{*}{ Soja de 2da } & $27,8 \%$ & 37,69 & 31,61 & $26.449,3$ \\
\hline & $127,8 \%$ & 173,1 & & $149.326,8$ \\
\hline
\end{tabular}

En función del tamaño de las actividades, la producción, los insumos, la dotación del capital, los precios de mercado de los granos al momento de cosecha y los precios de bienes y servicios, se estima el costo total, el cual incluye el costo de oportunidad explicitado en el método.

Dentro del costo de oportunidad, el rubro que mayor incidencia tiene es el capital Tierra. Si bien la tasa es relativamente baja $(4,0 \%)$, el valor de la tierra para la zona núcleo se ha incrementado considerablemente (2,5 veces) desde el 2004/05 hasta mediados de esta década.

Tabla $N^{\circ} 2$ Costo Total anual por campaña

CAMPAÑA 2015/2016

\begin{tabular}{lrr}
\cline { 2 - 2 } \multicolumn{1}{c}{ COSTOS TOTALES } & USD/año & \multicolumn{1}{c}{$\%$} \\
\hline Costos Variables & $73.639,9$ & $31,3 \%$ \\
\hline Gastos Fijos & $15.799,7$ & $6,7 \%$ \\
\hline Amortizaciones & $16.231,5$ & $6,9 \%$ \\
\hline Costo de Oportunidad Capital & $87.576,4$ & $37,2 \%$ \\
\hline Trabajo del Productor & $16.711,0$ & $7,1 \%$ \\
\hline Fondo de Prev. y Res. 12\% & $25.195,0$ & $10,7 \%$ \\
\hline \multicolumn{1}{c}{ Total } & $\mathbf{2 3 5 . 1 5 3 , 6}$ & $\mathbf{1 0 0 , 0 \%}$ \\
\hline
\end{tabular}

CAMPAÑA 2016/2017

\begin{tabular}{lrr}
\cline { 2 - 3 } \multicolumn{1}{c}{ COSTOS TOTALES } & USD/año & \multicolumn{1}{c}{$\%$} \\
\hline Costos Variables & $78.698,2$ & $32,4 \%$ \\
\hline Gastos Fijos & $14.582,3$ & $6,0 \%$ \\
\hline Amortizaciones & $15.613,1$ & $6,4 \%$ \\
\hline Costo de Oportunidad Capital & $92.018,1$ & $37,9 \%$ \\
\hline Trabajo del Productor & $15.822,4$ & $6,5 \%$ \\
\hline Fondo de Prev. y Res. 12\% & $26.008,1$ & $10,7 \%$ \\
\hline \multicolumn{1}{c}{ Total } & $\mathbf{2 4 2 . 7 4 2 , 1}$ & $\mathbf{1 0 0 , 0 \%}$ \\
\hline \multicolumn{2}{c}{} \\
\hline
\end{tabular}


CAMPAÑA 2017/2018

\begin{tabular}{|c|c|c|}
\hline COSTOS TOTALES & USD/año & $\%$ \\
\hline Costos Variables & $72.366,3$ & $30,9 \%$ \\
\hline Gastos Fijos & $14.841,7$ & $6,3 \%$ \\
\hline Amortizaciones & $15.419,8$ & $6,6 \%$ \\
\hline Costo de Oportunidad Capital & $90.968,7$ & $38,9 \%$ \\
\hline Trabajo del Productor & $15.276,8$ & $6,5 \%$ \\
\hline Fondo de Prev. y Res. $12 \%$ & $25.064,8$ & $10,7 \%$ \\
\hline Total & $233.938,1$ & $100,0 \%$ \\
\hline
\end{tabular}

CAMPAÑA 2018/2019

\begin{tabular}{|c|c|c|}
\hline COSTOS TOTALES & USD/año & $\%$ \\
\hline Costos Variables & $76.656,7$ & $33,4 \%$ \\
\hline Gastos Fijos & $12.065,1$ & $5,3 \%$ \\
\hline Amortizaciones & $14.909,5$ & $6,5 \%$ \\
\hline Costo de Oportunidad Capital & $88.496,1$ & $38,6 \%$ \\
\hline Trabajo del Productor & $12.520,3$ & $5,5 \%$ \\
\hline Fondo de Prev. y Res. $12 \%$ & $24.557,7$ & $10,7 \%$ \\
\hline Total & $229.205,5$ & $100,0 \%$ \\
\hline
\end{tabular}

CAMPAÑA 2019/2020

\begin{tabular}{lrr}
\cline { 2 - 2 } \multicolumn{1}{c}{ COSTOS TOTALES } & USD/año & \multicolumn{1}{c}{$\%$} \\
\hline Costos Variables & $81.439,9$ & $34,3 \%$ \\
\hline Gastos Fijos & $12.587,9$ & $5,3 \%$ \\
\hline Amortizaciones & $14.745,4$ & $6,2 \%$ \\
\hline Costo de Oportunidad Capital & $86.765,2$ & $36,5 \%$ \\
\hline Trabajo del Productor & $16.454,6$ & $6,9 \%$ \\
\hline Fondo de Prev. y Res. 12\% & $25.439,2$ & $10,7 \%$ \\
\hline \multicolumn{1}{c}{ Total } & $\mathbf{2 3 7 . 4 3 2 , 2}$ & $\mathbf{1 0 0 , 0 \%}$ \\
\hline
\end{tabular}

A partir del Ingreso y Costo total de la empresa y la superficie modal de la región se calcula la U.E.A. par ambas campañas.

Tabla $\mathrm{N}^{\circ} 3$ Superficie U.E.A. en ha por campaña

\begin{tabular}{lc} 
& USD/año \\
\cline { 2 - 2 } Ingresos & $144.951,6$ \\
\hline Egresos & $235.153,6$ \\
\hline & Ha/año \\
\hline U.EA. 2015/16 & $\mathbf{3 5 6 , 4}$ \\
\hline & \\
\cline { 2 - 2 } & USD/año \\
\cline { 2 - 2 } Ingresos & $135.419,8$ \\
\hline Egresos & $233.938,0$ \\
\hline & Ha/año \\
\hline U.EA. 2017/18 & $\mathbf{4 0 4 , 7}$ \\
\hline
\end{tabular}

\begin{tabular}{lc} 
& USD/año \\
\cline { 2 - 2 } Ingresos & $146.316,8$ \\
\hline Egresos & $242.742,1$ \\
\hline & Ha/año \\
\hline U.EA. 2016/17 & $\mathbf{3 7 2 , 7}$ \\
\hline & \\
\cline { 2 - 2 } & USD/año \\
\cline { 2 - 2 } Ingresos & $148.318,4$ \\
\hline Egresos & $229.205,0$ \\
\hline & Ha/año \\
\hline U.EA. 2018/19 & $\mathbf{3 1 6 , 0}$ \\
\hline
\end{tabular}

\begin{tabular}{lc}
\cline { 2 - 2 } Ingresos & USD/año \\
\cline { 2 - 2 } & $149.326,8$ \\
\hline Egresos & Ha/año \\
\hline & $\mathbf{3 4 2 , 3}$ \\
\hline U.EA. 2019/20
\end{tabular}


Gráfico $\mathrm{N}^{\circ}$ 1: Evolución de la U.E.A en ha por campaña

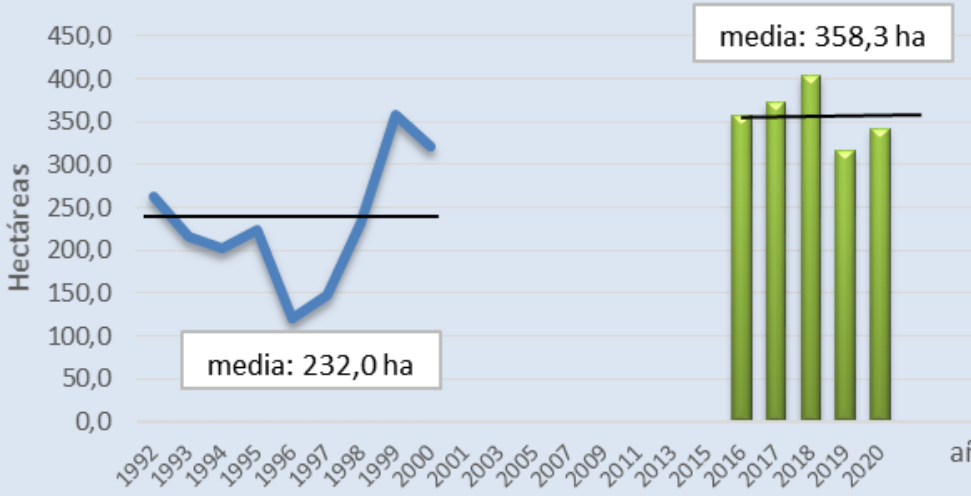

\section{CONCLUSIONES}

En primer lugar la superficie promedio de la U.E.A. (358,3 ha) de las campañas 2015/2016 (356,4 ha), 2016/2017 (372,7 ha), 2017/18 (404,1 ha), 2018/19 (316,0 ha) y 2019/2020 (342,3 ha) es significativamente superior a la superficie modal del área en estudio (sur de Santa Fe) que fue estimada en 135,4 ha. Esta situación desfavorable para las familias rurales explicaría en parte la persistente crisis económica y social que afecta al segmento de pequeños productores rurales, favoreciendo los procesos en los cuales abandonan su rol de productores cediendo la tierra en alquiler a otros productores con mayor capacidad empresarial, acentuando la concentración de la superficie en un número cada vez menor de empresas agropecuarias.

En segundo lugar si comparamos el promedio de la U.E.A. para el quinquenio $(\mathbf{3 5 8 , 3}$ ha) con el promedio oportunamente citado de la serie histórica 1992/2001 de $\mathbf{2 1 0 , 3}$ ha, se observa una incremento categórico. Esto estaría en parte explicado por el mayor valor de la tierra y su alta incidencia en el costo total, sumado a la implementación de los derechos de exportación, que desde inicios del 2002 han afectado el ingreso del productor en distinto grado, con una tendencia creciente. Durante el quinquenio el promedio de los derechos de exportación para la soja fue del 30\% y para trigo y maíz fueron eliminados en las primeras cuatro campañas, pero en la última fue reimplantado al 12\% para ambos cereales.

El valor promedio de la tierra en la serie 1992/2001 es 3.500,0 USD/ha, mientras que el valor del quinquenio es 14.079,0 USD/ha. El valor de la tierra incide como un costo de oportunidad cuando el productor es propietario y como un costo de producción directo cuando es arrendatario. Por lo tanto la U.E.A. es un indicador independiente de la tenencia de la tierra que se utiliza para caracterizar empresas que trabajan tierra en propiedad o en alquiler indistintamente. En este caso amerita un replanteo metodológico la utilización del valor de mercado del alquiler de la tierra, en remplazo de la utilización de una tasa de interés real fijada por el método oficial que varía entre el 5,0 y 4,0\%. Esta tasa no refleja el precio real del factor 
tierra, cuando la tasa de interés real de bajo riesgo es baja. En la última década las tasas reales de bajo riesgo son inferiores al 5-4\%, mientras que el valor de la tierra aumentó considerablemente.

Las diferencias en los valores de U.E.A. entre las campañas del quinquenio, obedecen principalmente a la combinación entre diferentes rendimientos de los cultivos por eventos climáticos y distintos precios de los granos. Esto se verifica en los valores de U.E.A. de las dos últimas campañas donde se da la combinación de altos rendimientos con mayores precios, arrojando los menores valores de U.E.A. del quinquenio.

Estos resultados indican la importancia de analizar el grado de evolución económica de la agricultura pampeana a través de la determinación de la U.EA. en una serie histórica de largo plazo permitiendo identificar y analizar los factores que mayor incidencia tienen en su determinación, así como brindar una explicación al creciente proceso de concentración de la tierra en la región pampeana. 


\section{BIBLIOGRAFÍA}

Bono G. (2013) "La reciente reglamentación cordobesa de la unidad económica ante la necesidad de una ley general de ordenamiento territorial con base en un criterio multidimensional de abordaje en la Ley Córdoba. Buenos Aires. a 30. nº 3 p. 237-252.

Censo Nacional Agropecuario (2008). INDEC - IPEC. Datos en

INDEC: Instituto Nacional de Estadística y Censos de la República Argentina

García Galilea M. (2016). La Unidad Económica Agropecuaria como herramienta para el ordenamiento territorial rural argentino. XII Congreso Español de Sociología. Datos en http://www.fes-sociologia.com/launidad-economica-agropecuaria-como-herramienta-para-el-ordenamient/congress-papers/2205/

González, M ; L, Paglietini (1993). Rentabilidad, Tasaciones y Tamaño Óptimo de la Empresa Agropecuaria. Editorial Agro Vet. Buenos Aires.

Ministerio de Agroindustria de la Nación (2020). Estimaciones Agrícolas. Datos en www.agroindustria.gob.ar/sitio/838reas/estimaciones/index.php

Porstmann, J.; Mancini, C.; Rivera Rúa, V.; Ercoli, R.; Wagner, A. Determinación de la Unidad Económica Agrícola en la región núcleo pampeana y su variación a lo largo del tiempo. XI Jornadas Interdisciplinarias de Estudios Agrarios y Agroindustriales Argentinos y Latinoamericanos. Buenos Aires, 2019.

Porstmann, J.; Ramírez, L; López G. y Guardatti S. (2013). Evaluación de los sistemas de producción de carne bovina intensiva en competencia con la agricultura. Ciencias Agronómicas - Revista XXII - Año 13 2013 / $027-034$.

Porstmann, Juan Carlos y López, Gabriela (2001). "Variaciones en la Unidad Agrícola Económica. La Influencia del Precio de los Granos". En: II Jornadas Interdisciplinarias de Estudios Agrarios y Agroindustriales, Buenos Aires.

Zuliani Susana (2017). "Unidad Económica Agropecuaria”. Material de Estudio de la Cátedra de Administración Rural. Facultad de Ciencias Agrarias. Universidad Nacional de Rosario. 